manuscripts supplements his famous edition of the Mathematical Papers. Cambridge University Press has just published the first volume of my edition of The Scientific Letters and Papers of James Clerk Maxwell.

On a rather broader front, the literature abounds with interpretative studies, of which C. Jungnickel and R. McCormmach's study of German physics, Intellectual Mastery of Nature (Chicago University Press, 1986), stands pre-eminent among recent publications. It would be a pity if physicists wanting to make use of historical work remained unaware of all this effort.

P. M. Harman is a visiting scholar in the Department of History of Science, Harvard University, Cambridge, Massachussetts 02138, USA.

\section{Holy grail or snark?}

\section{J. Ellis}

The Higgs Hunter's Guide: Frontiers in Physics. By J. F. Gunion, H. E. Haber, G. Kane \& S. Dawson. Addison-Wesley: 1990. Pp.425. £41.35, \$49.50.

WHAT is the most pressing problem in particle physics today? All confirmed experimental results agree dramatically with the Standard Model, giving us no clue how to progress beyond it. But the Standard Model is very cumbersome and inelegant, containing three distinct sets of gauge interactions, nine a priori arbitrary quark and lepton masses, four assorted mixing angles and phases to describe the charged-current weak interactions, and two other parameters thrown in by hand to break spontaneously the electroweak symmetry group. The latter 15 parameters all describe the couplings and mass of the mythical Higgs boson, supposed purveyor of masses to all the other elementary particles, but as yet únseen by experiment and the subject of furious theoretical debate. These seem reasons enough to cite the quest for the Higgs boson as the most pressing problem in particle physics, though some might cite the unification of all the interactions and understanding the proliferation of different particle types (the "flavour problem"). However, I consider the origin of mass and the mechanism of symmetry breaking prerequisites for addressing these important problems, particularly because the reasons for thinking that the Higgs boson should be accessible to present or planned accelerators are better than those for expecting detectable manifestations of unification or flavour physics.

With the recent start-up of the Large
Electron-Positron Collider (LEP) and advanced plans for the Large Hadron Collider (LHC) accelerator in Europe and the Superconducting Super Collider (SSC) in the United States, this book reviewing strategies for detecting the Higgs boson is therefore particularly timely. Moreover, the authors are among the best qualified to write on this important topic. Indeed, they provide the reader with a large amount of useful and accurate information about the expected properties of the Higgs boson and how to search for it in $\mathrm{e}^{+} \mathrm{e}^{-}$and hadron-hadron collisions. Nor is their attention focused only on the minimal Standard Model, because much of the book discusses nonminimal Higgs sectors, such as appear in supersymmetric and technicolour models. There are also discussions of axions, majorons and composite models. Also very useful are lengthy appendices containing detailed Feynman rules and formulae for Higgs decay rates. There is no doubt in my mind that this book should find a place in the office of any particle physicist interested in the problem of spontaneous symmetry breaking, which should include everyone working in highenergy physics.

I have only a few minor quibbles with the content of the book. It would have been good to have had a more extensive and elementary introduction to the motivation for spontaneous symmetry breaking in gauge theories and a general discussion of the Higgs mechanism. Some comparison and contrast with the theory of superconductivity could also have been appropriate, and might have made this excellent book more accessible to students and nonspecialists. Likewise, some discussion of the "naturalness" or "hierarchy" problem as a motivation for supersymmetric and technicolour models would also have been welcome.

As is perhaps inevitable in a topical book such as this, it has to some extent already been overtaken by events. Much of the discussion of a possible light Higgs boson has been rendered moot by the success of LEP at CERN, where just the Aleph experiment alone has already excluded any Standard Model Higgs boson weighing up to $41 \mathrm{GeV}$, and the combined data of all the four LEP experiments probably exclude the entire mass range up to $49 \mathrm{GeV}$. LEP has also given many limits on nonminimal Higgs bosons. By comparison, in their figure 1.1 , the authors predicted that LEP and the Stanford Linear Collider should be able to reach a mass of $30 \mathrm{GeV}$ only in 1993. Unfortunately, the SLC has been unable to muster sufficient luminosity to have any significant impact on the search for the Higgs boson.

These minor defects could easily be corrected in a second edition and do not detract from the great worth of this book, which will be an invaluable handbook until experiments find the Higgs boson or whatever replaces it, and probably long afterwards as well. This book will surely serve its avowed purpose of guiding the Higgs hunter towards her or his prey, and also interpreting whatever game she or he succeeds in bagging.

John Ellis is in the Theory Division, CERN, CH 1211 Geneva 23, Switzerland.

\section{Lucky dip}

\section{Stuart Sutherland}

Images and Understanding. Edited by Horace Barlow, Colin Blakemore and Miranda Weston-Smith. Cambridge University Press: 1990. Pp. 401. Hbk £40, $\$ 69.95$, pbk $£ 15, \$ 24.95$.

IN 1986 the Rank Prize Funds financed a conference on the use of images. Because I forgot to go, I can comment freely and without bias on the proceedings, now published somewhat tardily by Cambridge University Press under the title Images and Understanding.

The conference was nothing if not interdisciplinary for there were papers by psychologists, neurophysiologists, philosophers, computer scientists, and for good measure a writer, a theatre producer and a choreographer. Despite all the evidence refuting the belief, today even scientists seem to think that if enough people are gathered together, something good is bound to emerge. In this case what emerges is less a treasure trove than a lucky dip, which contains few pearls but has many trinkets whose glitter may amuse for a while.

Perhaps the most original contribution is that of John Willats who combines a practical knowledge of the visual arts with a formal training in engineering and a somewhat less formal one in psychology. $\mathrm{He}$ notes that paintings can attempt to represent surfaces either as seen from the observer's viewpoint or as they really are in 3-D space. Perspective is the prime example of the former category. An example of the latter is orthographic projection, in which selected surfaces are shown in their true physical shape, giving object-centred descriptions of at least part of the scene. Thus in P. Bonnard's picture, Nude in a Bath $T u b$, the top and one end of the bath are depicted in their true shapes, rather than in the shapes they would project to the retina. Willats also categorizes pictures and drawings by their denotational system, that is the rules or conventions for interpreting the marks in the picture. In a denotational system that is viewer-centred the brightness and colour of the marks reproduce an image on the retina that approximates as closely 
as possible to that which the scene would produce when viewed naturally. In other denotational systems, such as cartoons, a line may represent an edge: many other variations in brightness, for example those produced by shadows or by changes in albedo may be omitted. In some systems, hatching may arbitrarily represent a shadow. As Willats points out, such denotational systems have preprocessed the scene by making explicit the features that produce changes in brightness such as an edge or a shadow. These are novel and interesting ways of categorizing pictures, and they illuminate the motivation underlying different styles of painting.

Three other papers presented ideas and findings that were new - at least at the time of the conference. They were on colour (John Mollon), motion-detecting cells (Tony Movshon), and cells in the monkey's inferotemporal lobe that respond maximally to a person moving in a particular direction - backwards, forwards, left or right (David Perrett). Interestingly, some cells respond to directions of motion relative to the moving person's own position: one cell responded maximally to a person moving forwards to the right, but responded much less if he was facing left and walked backwards. If these results hold up, we are coming perilously close to grandmother cells. The interesting question is not, however, whether such cells exist, but how, if they do exist, they are wired up. The silence elicited by this problem is as profound as the problem itself.

To turn to the trinkets, the one that glitters most is a paper by Jon Darius on the fallibility of scientific observations, particularly in astronomy. It is well known that Martian canals were only disposed of by photographs from space craft, but he gives several other instances in which astronomers have collectively seen patterns that do not exist. One nice story concerns the map of Mercury that one astronomer compiled when it was thought that Mercury took the same period to rotate as to complete one orbit, thus always presenting the same face to the observer. Although it is now known that these periods are quite different, three other astronomers, each looking at a different face, drew maps that were almost identical to the original one.

Of the other papers, two may be singled out for their amusement value. John Krebs recounts the behaviour of the honeyguide, an African bird that is adept at locating bees' nests but rather poor at obtaining access to them in order to eat the honey. It has solved the problem by leading human hunters to the nests it has located: when the hunters open them up, it is able to gorge itself. Mike Land introduces us to the scallop's unusual method of forming a retinal image - by a concave mirror behind the retina - and the even more bizarre method used by the shrimp - a series of flat mirrors in front of the retina all of which send light from the same point in space to the same point on the retina: he cites several similar curiosa. Entertaining though such discoveries may be, they are hardly likely to change the way scientists think.

In fact, there are few theories, whether grand or small, to be found in this collection. Too many of the authors tell us, often in some detail, what we already know. Colin Blakemore puzzles over the question of why visual and other sensory maps exist, but misses the obvious answer that normally it is necessary to analyse the retinal image locally. The parts of an object are contiguous and must be processed together. In a different world, it might have been necessary to put together the pattern of light falling in the top righthand corner of the retina with that in the bottom left, which would have needed a form of mapping quite unlike existing cortical maps.

The book has two curious features. The first is what it leaves out. There is nothing

\section{Pretty pictures \\ P. T. Saunders}

Envisioning Information By Edward R. Tufte. Graphics Press: 1990. Pp. 126. $\$ 48$.

ONE picture, so we are told, is worth a thousand words. Certainly most scientists seem to think so, judging by the large number of figures and tables we put into our papers. With pictures even more than with words, however, it is not always easy to convey information in a way that is both attractive and clear, especially because in both cases the medium is less complex and dynamic than the message it is being asked to carry.

In Envisioning Information, Edward Tufte discusses how pictures can be made more effective. But the book is not a text on graphic design. Its aim is to illustrate general principles that help to identify and explain design excellence, not to teach specific techniques. Nor is it aimed specifically at the scientist. There are, to be sure, examples from science, but more from other fields. But they have been chosen with a view to holding the reader's interest, and almost anyone is bound to come across something that he did not know before and is likely to remember.

Tufte provides many beautiful examples of "cognitive art" - and a few horrors as well, to show what can happen when the principles are ignored. The illustrations include such gems as diagrams from an 1847 colour edition of Euclid's Elements in which the figures resemble paintings by Mondrian. And even though the focus on the difficulty of interpreting medical $\mathrm{X}$ rays or the electrocardiogram nor on the merits of attempting to do so by computer. Nor is there anything on the bizarre disruption of images that can occur in braindamaged patients. Dreams and hallucinations receive no mention, and apart from a philosophical contribution there is little on the nature of mental images. Second, the individual papers have remarkably little relevance to one another. It is just possible that some of the participants will be inspired by the contributions of workers in other disciplines to change their vicw of their own patch, but it seems unlikely.

Most of the papers are clear and some are elegant: few are deep. They will appeal less to the scientist than to the general reader, whose delight in trinkets is unaffected by their antiquity. I half wish I had remembered to attend the conference.

Stuart Sutherland is at the University of Sussex, Laboratory of Experimental Psychology, Brighton, BN1 9QG, UK.

of the book is on graphics, the text is especially well written.

All the same, Envisioning Information is likely to disappoint any scientist who buys it chiefly in the hope of learning how to write better papers. After reading it, I went through a recent issue of Nature looking for figures that I thought would have been better if the authors had followed some of Tufte's suggestions, and I did not find any. That may reveal a lack of imagination on my part, but I think it also indicates that most figures in scientific journals are pretty straightforward. Most of the graphs looked clear enough to me, and I doubt there is very much that even Tufte could do with a photograph of a gel.

A particular shortcoming from the point of view of the scientist is that much of the book is concerned with the effective use of colour. This is an important topic, and it makes the book itself more pleasing to look at, but it is of little practical value for authors who are almost always restricted to black and white on grounds of cost.

Envisioning Information is not as relevant for the scientist as Tufte's earlier The Visual Display of Quantitative Information. You would not want to keep it on the laboratory shelf next to handbooks of constants, tables of integrals and style manuals. On the other hand, I enjoyed reading it, it did teach me something about the use of graphics - and if it had been a more practical text I would not have learned anything at all because I would never have picked it up in the first place.

P. T. Saunders is in the Department of Mathematics, King's College, The Strand, London, UK. 